\title{
Left ventricular pacing site and timing optimization during biventricular pacing using a multielectrode patch in pigs
}

George Berberian, MD, ${ }^{\text {a }}$ T. Alexander Quinn, MS, ${ }^{\text {b }}$ Santos E. Cabreriza, MBA, a Jon-Emile S. Kenny, BS, Cara A. Garofalo, MD, ${ }^{c}$ Alan D. Weinberg, MS, ${ }^{d}$ and Henry M. Spotnitz, MD ${ }^{a, *}$

From the Departments of Surgery, ${ }^{a}$ Biomedical Engineering, ${ }^{\mathrm{b}}$ Pediatrics, ${ }^{\mathrm{c}}$ and Biostatistics, ${ }^{\mathrm{d}}$ Columbia University, College of Physicians and Surgeons, New York, NY.

Supported in part by the National Heart, Lung and Blood Institute of the National Institutes of Health (RO1 HL 48109 to Dr Spotnitz) and in part by the Department of Surgery, Columbia University College of Physicians and Surgeons.

Received for publication Sept 21, 2006; revisions received March 11, 2007; accepted for publication April 25, 2007.

Address for reprints: Henry M. Spotnitz, MD, Department of Surgery, Columbia College of Physicians and Surgeons, 622 West 168th St, PH 14-103, New York, NY 10032 (E-mail: hms2@columbia.edu).

*George H. Humphreys, II Professor of Surgery.

J Thorac Cardiovasc Surg 2007;134:574-8

$0022-5223 / \$ 32.00$

Copyright $\odot 2007$ by The American Association for Thoracic Surgery

doi:10.1016/j.jtcvs.2007.04.050
Objectives: Biventricular pacing is important therapy for congestive heart failure, reversing left ventricular dysfunction in dilated cardiomyopathy. Although left ventricular lead location and right ventricular-left ventricular delay are believed to be critical in biventricular pacing, there is no established technique for optimizing pacing site and timing.

Methods: After median sternotomy in 8 anesthetized pigs, an ultrasonic flow probe was placed on the ascending aorta to measure cardiac output, and pressure catheters were inserted into both ventricles. Temporary bipolar epicardial pacing leads were attached to the right atrium and anterior right ventricle. A patch with 5 bipolar electrodes was placed behind the left ventricle. A temporary bipolar lead was also placed on the left ventricular apex. Complete heart block was established by ethanol ablation. Right ventricular pressure overload was induced by snaring the pulmonary artery until right ventricular systolic pressure doubled. Dual-chamber mode biventricular pacing was instituted at 9 right ventricular-left ventricular delays, $+80 \mathrm{~ms}$ to $-80 \mathrm{~ms}$ in $20 \mathrm{~ms}$ increments, and 6 left ventricular sites. Data from the 54 combinations of these variables were acquired in a randomized fashion. Mixed model technology was used for statistical analysis.

Results: Qualitatively, two unique site/timing pairs were optimal. Statistically, pacing the obtuse margin at a right ventricular-left ventricular delay of $+60 \mathrm{~ms}$ (mean cardiac output $=1.80 \mathrm{~L} / \mathrm{min}$ ) and the inferolateral wall at a right ventricularleft ventricular delay of $-20 \mathrm{~ms}$ (mean cardiac output $=1.79 \mathrm{~L} / \mathrm{min}$ ) was superior to all other site/timing combinations (mean cardiac output $=1.71 \mathrm{~L} / \mathrm{min} ; P=.006$ ).

Conclusions: Left ventricular pacing site and right ventricular-left ventricular delay can be optimized with a multielectrode patch and randomized data collection. This technique can be used further in clinical studies.

I ncreasing clinical evidence suggests that biventricular pacing (BiVP) can be beneficial for patients with congestive heart failure (CHF), right and/or left bundle branch block, and long QRS complexes. Three important clinical studies (InSync, MUSTIC, and MIRACLE) have demonstrated a benefit to patients with $\mathrm{CHF}$ as evidenced by improved hemodynamics, quality of life, and decreased hospital admission owing to CHF. ${ }^{1-3}$ However, many patients did not respond to BiVP in these trials, suggesting that better techniques of patient selection and pacing optimization are needed. Adjustment of atrioventricular delay, right ventricular-left ventricular delay (RLD), and ventricular pacing site may improve ventricular synchrony and increase cardiac output (CO) and ventricular mechanics during BiVP.

Median sternotomy provides access to the ascending aorta and pulmonary artery, allowing measurement of flow velocity by ultrasonic transit-time flow probes. Transit-time ultrasound has been shown to be an accurate, continuous method of 


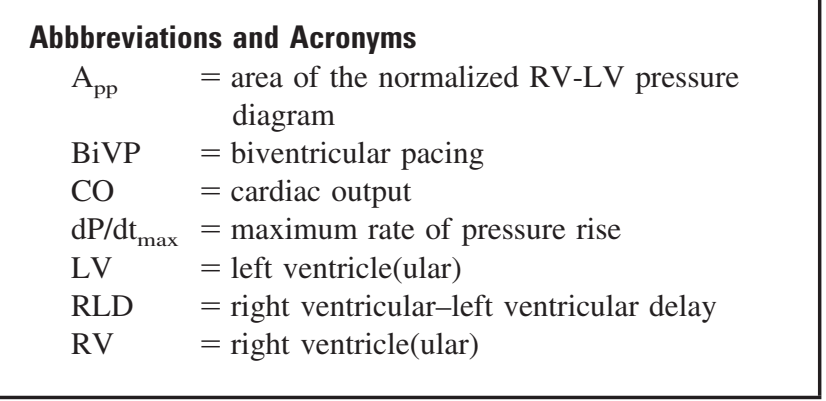

measuring flow and has been validated against a right heart bypass preparation. ${ }^{4}$ These flow velocities can be integrated to provide a digital readout of $\mathrm{CO}$.

Open-chest animal models have been used to assess effects of pacing strategies on left ventricular (LV) contractility. Prinzen and associates ${ }^{5}$ evaluated the effect of pacing strategies on cardiac function in a canine model using rate of pressure rise $(\mathrm{dP} / \mathrm{dt})$ and stroke volume as indices of $\mathrm{LV}$ synchrony. To date, there has been no systematic study of the effect of LV pacing site and timing during BiVP. We hypothesized that during BiVP, a multielectrode patch placed in the posterior pericardium could allow for rapid testing of multiple LV sites and RLD and could identify effects of LV site and RLD on CO and other measures of function.

\section{Materials and Methods}

All animals received humane care in compliance with the "Principles of Laboratory Animal Care" developed by the Institute of Laboratory Animal Resources and the "Guide for the Care and Use of Laboratory Animals" prepared by the Institute of Laboratory Animal Resources and published by the National Institutes of Health (NIH Publication number 85-23, revised 1985).

\section{Surgical Preparation}

Eight domestic pigs (40-45 kg) were anesthetized with ketamine hydrochloride ( $20 \mathrm{mg} / \mathrm{kg}$ intramuscularly), xylazine hydrochloride $(0.5 \mathrm{mg} / \mathrm{kg}$ intramuscularly), and atropine sulfate $(2 \mathrm{mg} / \mathrm{kg}$ intramuscularly). Pigs were intubated and mechanically ventilated, with arterial blood gas values being maintained within physiologic norms. Anesthesia was maintained with inhalation isofluorine $(1.5 \%-2 \%)$ in oxygen. An 18-gauge angiocatheter was placed in an ear vein for an intravenous infusion of $0.9 \%$ saline. Electrocardiogram leads were attached to the limbs, and the left femoral artery was instrumented with a 20-gauge angiocatheter attached to a pressure transducer to measure arterial pressure. After median sternotomy and longitudinal pericardiotomy, a lidocaine bolus ( 3 $\mathrm{mg} / \mathrm{kg}$ intravenously) was given and a lidocaine drip started at 50 $\mu \mathrm{g} \cdot \mathrm{kg}^{-1} \cdot \min ^{-1}$ to suppress arrhythmias. A $24-\mathrm{mm}$ real-time ultrasonic flow probe (Transonic Systems Inc, Ithaca, NY) was placed on the ascending aorta. Temporary bipolar epicardial pacing leads (Medtronic, Inc, Minneapolis, Minn) were placed on the right atrium, anterior surface of the right ventricle (RV), and apex of the LV. A multielectrode patch with 5 bipolar electrodes (Figure

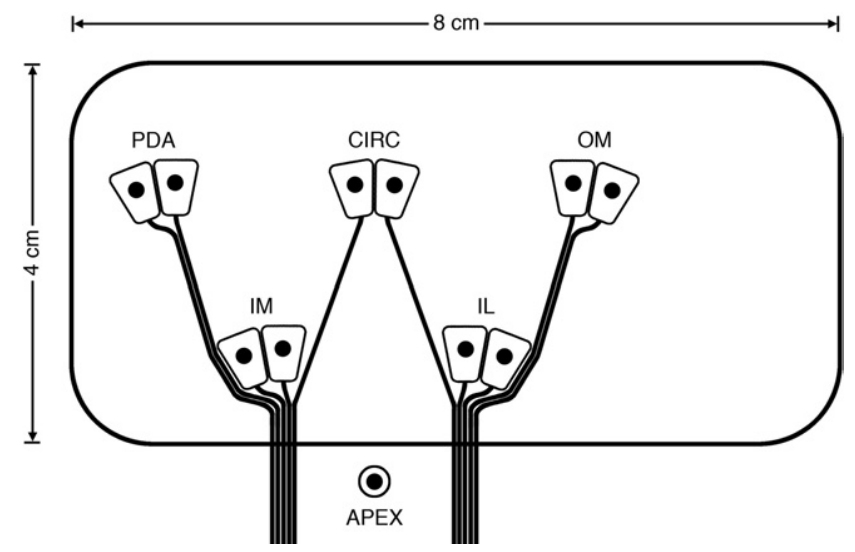

Figure 1. Diagram of the multielectrode patch with 5 bipolar electrodes and corresponding left ventricular sites. Dimensions are indicated in centimeters. Apical leads were sewn in. APEX, Apical; CIRC, circumflex; IL, inferolateral; IM, inferomedial; OM, obtuse margin; PDA, posterior descending.

1), corresponding to $5 \mathrm{LV}$ sites, was placed in the posterior pericardium around the LV (Figure 2). Pericardial stay sutures were placed and the pericardium was wrapped around the patch to secure it in place. Pacing wires were connected to a custom-housed InSync III pacemaker (Medtronic) to allow temporary pacing with variable RLD.

Once proper function of the pacemaker leads was confirmed, complete heart block was established by injection of $0.5-\mathrm{mL}$ aliquots of $100 \%$ ethanol into the region of the bundle of His at the base of the aorta. The cumulative amount of ethanol required to establish complete heart block ranged from 0.5 to $1 \mathrm{~mL}$. Animals were heparinized with $300 \mathrm{IU} / \mathrm{kg}$ intravenously, and calibrated $5 \mathrm{~F}$ micromanometer catheters (Millar Instruments, Inc, Houston, Tex) were placed in both the RV and LV through purse-string sutures. $\mathrm{RV}$ pressure overload was induced by snaring the pulmonary artery with umbilical tape until RV systolic pressure doubled.

Atrial tracking dual-chamber mode BiVP was initiated at a heart rate of 90 beats/min with an atrioventricular delay of $150 \mathrm{~ms}$ in all animals. Animals were paced at 9 RLDs, $+80 \mathrm{~ms}$ (RV-first pacing) to $-80 \mathrm{~ms}$ (LV-first pacing) in $20-\mathrm{ms}$ increments, and 6 LV sites (apex, inferomedial, inferolateral, posterior descending, circumflex, obtuse margin) for 30-second intervals. Data from the 54 possible combinations were acquired in a randomized fashion. Animals were humanely killed at the conclusion of the experiment.

\section{Data Analysis}

Analog data for electrocardiogram, arterial pressure, RV pressure, LV pressure, and aortic flow velocity were sampled and transferred through a 16-channel analog-to-digital converter (ADInstruments, Inc, Milford, Mass) to a personal computer (Apple Computer, Cupertino, Calif). CO was determined for each experimental setting by integrating aortic flow velocity over time during a single complete respiratory cycle free of arrhythmia within each 30second interval. In 7 pigs, ventricular systolic function was assessed from the same cardiac cycles by $\mathrm{RV}$ and $\mathrm{LV} \mathrm{dP} / \mathrm{dt}_{\max }$. 


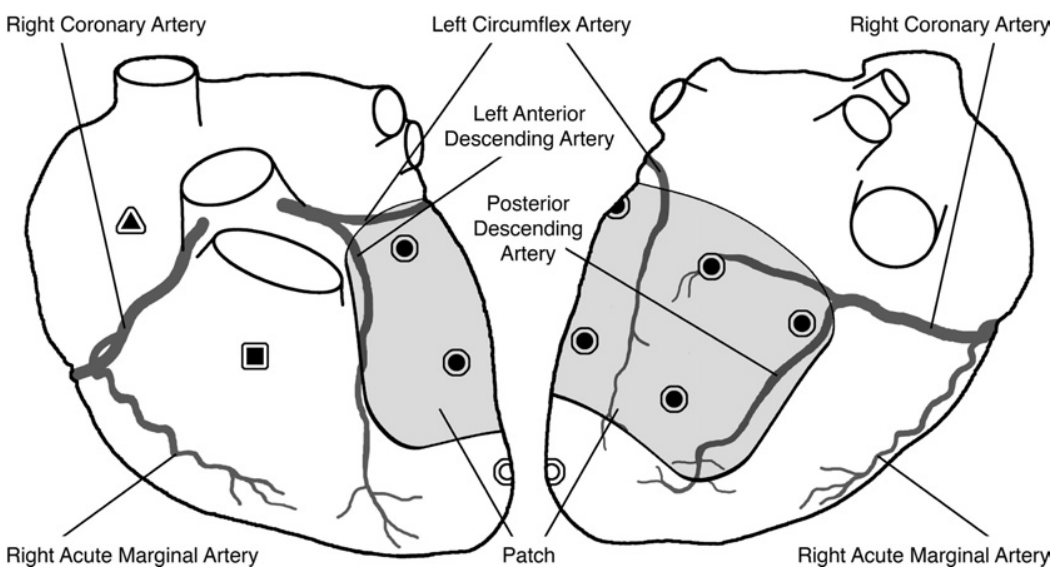

Figure 2. Diagram of the anatomic location of the multielectrode patch and contained electrodes on the left ventricle (dark circles). Pacing leads were also placed on the apex (open circle), anterior surface of the right ventricle (square), and right atrium (triangle).

Mechanical interventricular synchrony was quantified by the area of the normalized RV-LV pressure diagram $\left(\mathrm{A}_{\mathrm{PP}}\right){ }^{6} \mathrm{~A}_{\mathrm{PP}}$ expresses synchrony based on the pressure during the complete cardiac cycle, with a loop area of zero indicating complete synchrony and a maximum area of one indicating complete asynchrony. A counterclockwise loop results in positive values, indicating earlier RV than LV pressure generation.

The resulting data were imported into Matlab (The MathWorks, Inc, Natick, Mass). The percentage change in $\mathrm{CO}$ from the mean value of all settings was calculated in each pig. The values for each combination of settings were averaged across the group. Results were visualized by use of response surfaces with LV pacing site on the ordinate, RLD on the abscissa, and magnitude represented by a color map linearly interpolated between measured values. Contour lines represent incremental changes. For surfaces of $\mathrm{CO}$ and $\mathrm{dP} / \mathrm{dt}_{\text {max }}$, the highest values are represented by red, and for surfaces of $A_{P P}$, complete synchrony $\left(A_{P P}=0\right)$ is represented by white.

\section{Statistical Methods}

LV site/RLD combinations were modeled in SAS via the PROC MIXED procedure for repeated measurements (SAS Institute, Inc., Cary, NC). Owing to the fact that repeated measurements within animals may be correlated, this procedure allows one to model this "correlation structure" commonly referred to as a covariance pattern. This accurate estimate will allow for estimates of the standard errors of measurement and, therefore, more powerful tests. There are a number of various covariance structures to choose from. Three of the more common covariance structures include "compound symmetry," for correlations that are constant for any two points in time, "auto-regressive order one," for correlations that are smaller for time points further apart, and "unstructured," which has no mathematical pattern within the covariance matrix. The compound symmetry structure provided the best fit.

\section{Results}

Figure 3 shows a representative plot of beat-to-beat $\mathrm{CO}$ measurements over 27 minutes of continuous data collection during 1 experiment. Figure 4 is a response surface plot demonstrating percentage change from the mean $\mathrm{CO}$ for all possible
LV site/RLD combinations averaged over all 8 experiments. Qualitatively, 2 unique LV site/RLD pairs were optimal. Pacing the obtuse margin at an RLD of $+60 \mathrm{~ms}$ (mean $\mathrm{CO}=1.80$ $\mathrm{L} / \mathrm{min}$ ) and the inferolateral wall at an RLD of $-20 \mathrm{~ms}$ (mean CO $=1.79 \mathrm{~L} / \mathrm{min}$ ) was superior to all other $\mathrm{LV}$ site/RLD combinations (mean $\mathrm{CO}=1.71 \mathrm{~L} / \mathrm{min} ; P=.006$ ). Figure 5 shows the average hemodynamic effects of LV pacing site and RLD averaged over 7 experiments using response surface plots. LV dP/ $\mathrm{dt}_{\max }$ increased $14 \%$ with negative RLD, reaching a maximum and plateauing around $-20 \mathrm{~ms}$. $\mathrm{RV} \mathrm{dP} / \mathrm{dt}_{\max }$ increased $30 \%$ and $\mathrm{A}_{\mathrm{PP}}$ decreased to near synchronous with positive RLD, plateauing around $+40 \mathrm{~ms}$. LV pacing site only affected $\mathrm{LV} \mathrm{dP} / \mathrm{dt}_{\max }$, with sites near the apex producing the highest values.

\section{Discussion}

Recent clinical studies of endocardial BiVP differ in suggesting that cardiac function is maximized by localization of $\mathrm{LV}$ pacing leads in the midlateral region of the $\mathrm{LV}^{7,8}$ or

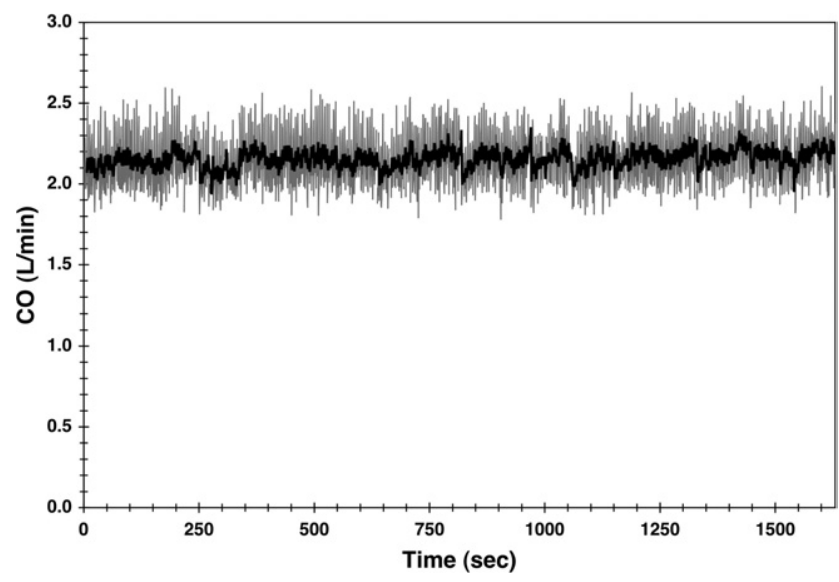

Figure 3. A representative plot of beat-to-beat cardiac output measurements over 27 minutes of continuous data collection during 1 experiment. CO, Cardiac output. 


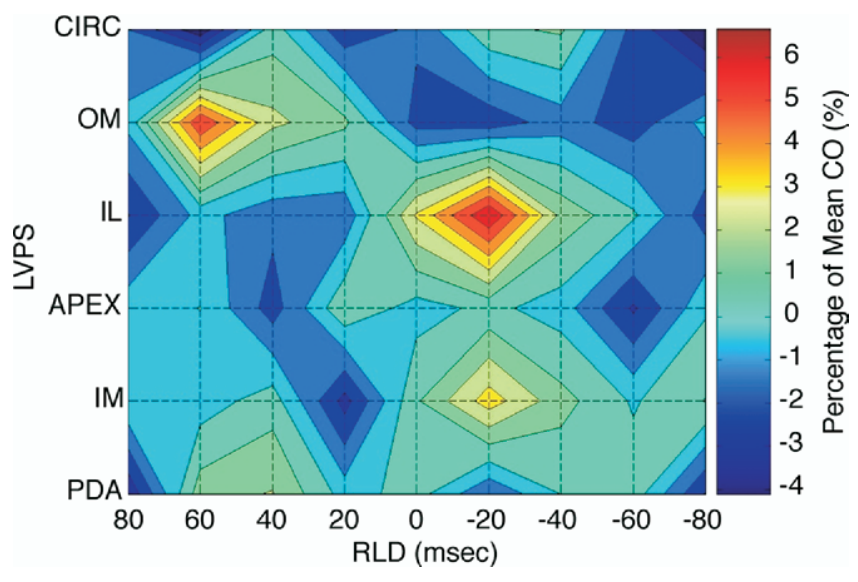

Figure 4. Surface plot demonstrating percentage change from mean cardiac output for all possible left ventricular pacing site/right-left ventricular delay combinations averaged over 8 experiments. Contour lines indicate a $1 \%$ change in cardiac output. The highest values are represented by red. APEX, Apical; CIRC, circumflex; CO, cardiac output; IL, inferolateral; IM, inferomedial; LVPS, left ventricular pacing site; OM, obtuse margin; PDA, posterior descending artery; $R L D$, right ventricular-left ventricular delay.

other locations. ${ }^{9,10}$ Endocardial LV lead position is often limited by anatomy of the cardiac veins. Many locations are inaccessible, resulting in implantation failure in $5 \%$ to $14 \%$ of attempts. ${ }^{11,12}$

Dekker and associates ${ }^{13}$ showed that optimal epicardial LV pacing site acutely increased LV stroke volume, dP/ $\mathrm{dt}_{\max }$, and ejection fraction relative to baseline, whereas suboptimal sites did not change or worsened LV function. The InSync III trial showed that $77 \%$ of patients had an optimal RLD other than $0 \mathrm{~ms}$. Optimization acutely increased echocardiographic-Doppler LV stroke volume by
$11.3 \%$ in these patients, and $17 \%$ of these patients experienced $20 \%$ or greater improvement in stroke volume. ${ }^{14}$

The present study sought to develop techniques that could be used clinically for optimization of BiVP during epicardial implantation. A multielectrode patch placed in the posterior pericardium allowed for rapid testing of multiple LV sites, avoiding mechanical effects of lead manipulation. Changing both site and RLD simultaneously and in randomized fashion avoids linear effects of one site-timing combination on the next and also eliminates possible bias of the investigators on outcome.

We have used $\mathrm{CO}$ as the dependent variable in our BiVP optimization studies, because $\mathrm{CO}$ is a fundamental determinant of organ perfusion. However, many other variables have been used clinically for BiVP optimization, including echocardiographic studies of posterior wall motion and ventricular synchrony. Because $\mathrm{CO}$ depends on multiple variables and requires time to stabilize, determinants of $\mathrm{CO}$ could ultimately prove more valuable for rapid optimization. These determinants could include echocardiographic indices, $\mathrm{A}_{\mathrm{PP}}$, or even shortening of $\mathrm{QRS}$ duration.

Previous studies from our laboratory have demonstrated that in a similar model of severe pressure overload, CO was maximized with an RLD of $+40 \mathrm{~ms}$ (RV-first pacing). ${ }^{15}$ In that experiment, the LV was paced at only one site, the obtuse margin. In the present study, multiple LV sites were tested and two optimal LV site/RLD combinations were identified: obtuse margin/RLD +60 ms and inferolateral/RLD $-20 \mathrm{~ms}$. Our laboratory is in the process of defining mechanisms for the effects of RLD on CO. ${ }^{16}$ In a recent study of $\mathrm{LV}$ and $\mathrm{RV}$ pressure development in our experimental model of RV pressure overload, optimized pacing was associated with statistically significant benefits in $\mathrm{RV} \mathrm{dP} / \mathrm{dt}_{\max }$ and interventricular synchrony. ${ }^{17}$ In the present study of RV failure, RV function and ven-
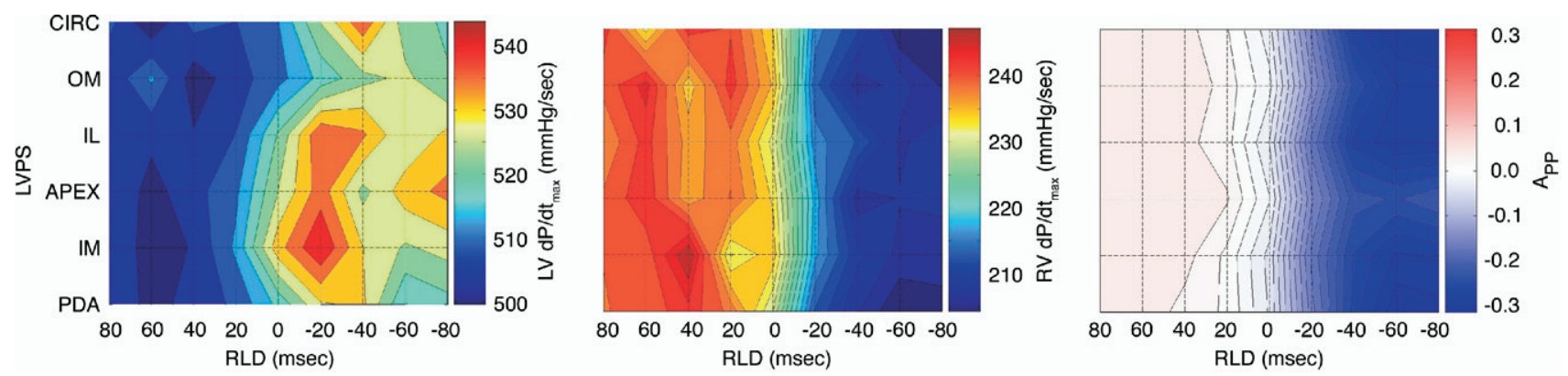

Figure 5. Response surfaces relating left and right ventricular $\mathrm{dP} / \mathrm{dt}_{\max }$ and the area of the normalized right ventricular-left ventricular pressure diagram, $A_{P P}$, to all possible left ventricular pacing site/right ventricular-left ventricular delay combinations averaged over 7 experiments. Contour lines represent $1 \%$ changes in measured values. For surfaces of $\mathrm{dP} / \mathrm{dt}_{\text {max }}$, the highest values are represented by red, and for surfaces of $A_{P P}$, complete synchrony $\left(A_{P P}=0\right)$ is represented by white. $A P E X$, Apical; $A_{P P}$, area of the normalized right ventricular-left ventricular pressure diagram; $C I R C$, circumflex; $d P / d t_{\text {max }}$, maximum rate of pressure rise; IL, inferolateral; IM, inferomedial; $L V$, left ventricle; $O M$, obtuse margin; $P D A$, posterior descending artery; $R V$, right ventricle. 
tricular interaction were optimized with an RLD of +40 $\mathrm{ms}$ at all LV sites.

This study demonstrates an epicardial BiVP optimization technique applicable to permanent pacing in patients with chronic heart failure and also to temporary pacing in acute heart failure after cardiac surgery. In fact, given the anomaly of publication delays, we ${ }^{18}$ have already applied and published the method developed in the present study in a single patient with dilated cardiomyopathy. Results in that clinical study demonstrate effects of site and timing on $\mathrm{CO}$ that cover a range of $70 \%$, considerably larger than the $10 \%$ variation observed here. This reflects inherent limitations of our models of $\mathrm{RV}$ and $\mathrm{LV}^{19}$ failure and emphasizes the need for a model that manifests advanced LV failure and QRS prolongation resulting from impaired conduction velocity.

However, despite possibly limited clinical relevance, our studies have provided an important opportunity to develop clinically relevant methodology, a team to apply these methods intraoperatively, and improved understanding of the pathophysiology of BiVP.

Optimization of LV pacing site and RLD using a multielectrode patch and randomized data collection provides an innovative, objective, and intuitive tool for solving a critical problem in BiVP. Application of this method in patients can provide unique information that improves clinical results of pacing for heart failure.

\section{References}

1. Gras D, Leclercq C, Tang AS, Bucknall C, Luttikhuis HO, KirsteinPedersen A. Cardiac resynchronization therapy in advanced heart failure the multicenter InSync clinical study. Eur J Heart Fail. 2002; 4:311-20.

2. Cazeau S, LeClerq C, Lavergne T, Walker S, Varma C, Linde C, et al. Effects of multi-site biventricular pacing in patients with heart failure and intraventricular conduction delay. N Engl J Med. 2001;344:873-80.

3. Abraham WT, Fisher WG, Smith AL, Delurgio DB, Leon AR, Loh E, et al. Cardiac resynchronization in chronic heart failure. $N$ Engl J Med. 2002;346:1845-53.

4. Dean DA, Chao-Xiang J, Cabreriza S, D'Alessandro DA, Spotnitz, HM. Validation study of a new transit time ultrasonic flow probe for continuous great vessel measurements. ASAIO J. 1996;42:M671-6.

5. Prinzen FW, van Oosterhout MFM, Vanagt WYR, Storm C, Reneman RS. Optimization of ventricular function by improving the activation sequence during ventricular pacing. Pacing Clin Electrophysiol. 1998; 21(11 Pt 2):2256-60.

6. Yu Y, Kramer A, Spinelli J, Ding J, Hoersch W, Auricchio A. Biventricular mechanical asynchrony predicts hemodynamic effect of uni- and biventricular pacing. Am J Physiol Heart Circ Physiol. 2003;285:H2788-96.

7. Auricchio A, Klein H, Tockman B, Sack S, Stellbrink C, Neuzner J, et al. Transvenous biventricular pacing for heart failure: can the obstacles be oversome? Am J Cardiol. 1999;83(5B):136D-42D.

8. Butter C, Auricchio A, Stellbrink C, Schlegl M, Fleck E, Horsch W, et al. Should stimulation site be tailored in the individual heart failure patient? Am J Cardiol. 2000;86(9 Suppl 1):144K-51K.

9. Pappone C, Rosanio S, Oreto G, Tocchi M, Gulletta S, Salvati A, et al. Cardiac pacing in heart failure patients with left bundle branch block: impact of pacing site for optimizing left ventricular resynchronization. Ital Heart J. 2000;1:464-9.

10. Ansalone G, Giannantoni P, Ricci R, Trambaiolo P, Fedele F, Santini M. Doppler myocardial imaging to evaluate the effectiveness of pacing sites in patients receiving biventricular pacing. $J$ Am Coll Cardiol. 2002;39:489-99.

11. Alonso C, Leclercq C, d'Allonnes FR, Pavin D, Victor F, Mabo P, et al. Six year experience of transvenous left ventricular lead implantation for permanent biventricular pacing in patients with advanced heart failure: technical aspects. Heart. 2001;86:405-10.

12. Valls-Bertault V, Mansourati J, Gilard M, Etienne Y, Munier S, Blanc JJ. Adverse events with transvenous left ventricular pacing in patients with severe heart failure: early experience from a single centre. $E u$ ropace. 2001;3:60-3.

13. Dekker LAJ, Phelps B, Dijkman B, van der Nagel T, van der Veen FH, Geskes GG, et al. Epicardial left ventricular lead placement for cardiac resynchronization therapy: optimal pace site selection with pressurevolume loops. J Thorac Cardiovasc Surg. 2004;127:1641-7.

14. Leon AR, Abraham WT, Brozena S, Daubert JP, Fisher WG, Gurley $\mathrm{JC}$, et al. Cardiac resynchronization with sequential biventricular pacing for the treatment of moderate-to-severe heart failure. $\mathrm{J} \mathrm{Am} \mathrm{Coll}$ Cardiol. 2005;46:2298-304.

15. Rabkin DG, Cabreriza SE, Curtis LJ, Mazer SP, Kanter JP, Weinberg $\mathrm{AD}$, et al. Load dependence of cardiac output in biventricular pacing: right ventricular pressure overload in pigs. J Thorac Cardiovasc Surg. 2004; 127:1713-22.

16. Rabkin DG, Cabreriza SE, Curtis LJ, Quinn TA, Weinberg AD, Hordof AD, et al. Mechanisms of optimized biventricular pacing in pulmonary stenosis: effects on left ventricular geometry in swine. Pacing Clin Electrophysiol. 2004:27:1060-71.

17. Quinn TA, Berberian G, Cabreriza SE, Maskin LJ, Weinberg AD, Holmes JW, et al. Effects of sequential biventricular pacing during acute right ventricular pressure overload. Am J Physiol Heart Circ Physiol. 2006;291:H2380-7.

18. Berberian G, Cabreriza SE, Quinn TA, Garofalo CA, Spotnitz HM. Left ventricular pacing site-timing optimization during biventricular pacing using a multi-electrode patch. Ann Thorac Surg. 2006; 82:2292-4

19. Berberian G, Quinn TA, Cabreriza SE, Garofalo CA, Barrios DM, Weinberg AD, et al. Load dependence of cardiac output in biventricular pacing: left ventricular volume overload in pigs. $J$ Thorac Cardiovasc Surg. 2006;131:666-70. 